\title{
Live, eat, sleep football? Body size and composition and beliefs about physical activity in an international cohort of school-aged boys participating in regular team sport
}

\author{
K. H. Hart ${ }^{1}$, L. Boyle ${ }^{1}$, C. A. Hartwick ${ }^{2}$, L. Moreno ${ }^{3}$, S. A. Lanham-New ${ }^{1}$ and B. Fielding ${ }^{1}$ \\ ${ }^{1}$ Department of Nutritional Sciences, Faculty of Health and Medical Sciences, University of Surrey, Guildford GU2 \\ $7 X H,{ }^{2}$ Danone Institute International, Paris Descartes University, Palaiseau, France and ${ }^{3}$ University of Zaragoza, \\ Zaragoza, France
}

Physical inactivity is identified as the fourth leading risk factor for global mortality ${ }^{(1)}$ yet despite this, and the development of clear activity recommendations for all ages including children, healthful activity habits are failing to be established in childhood. Fewer than 1 in 4 children in OECD countries report that they undertake regular moderate-to-vigorous exercise ${ }^{(2)}$ and participation in team sports may be specifically in decline despite the potential benefits, which extend beyond physical health. The Danone Nations Cup (DNC) is an annual football tournament and nutrition education programme involving 32 nations which focuses on the promotion of "an inspiring and healthy future". This study aimed to undertake a cross-country comparison of body size and composition, activity levels and beliefs in children who are engaged in team sport to better understand its role in promoting healthy lifestyles.

The 2013 DNC final took place in England and participating children completed questionnaires at the beginning and end of the week-long event, with self-report of habitual food intake and activity levels and knowledge of general nutrition and activity recommendations. Subject to parental informed consent and child assent anthropometric measurements (weight, height, body fat via bioelectric impedance and waist and hip circumferences) were also taken on one occasion using standard paediatric methodologies.

Pre-questionnaires were completed by 190 children (sample A, 16 countries) and anthropometric data was collected from 183 children (sample B, 16 countries, all male, mean age 12.3[0.4] years). Fifty two percent of the children in sample A ( $n=93$ ) used active transport to school and $73 \%(n=139)$ were active during school breaks. Sixty six percent $(n=125)$ reported 5 or more hours of sport per week but only $23 \%(n=43)$ cited the recommendation for children of their age to be active for at least one hour per day with over a third $(n=61,35 \%)$ believing the recommendations did not include daily activity. Modal screen time was 30 minutes per day $(n=46$, $24 \%)$. The majority of the children (57\%) reported having televisions but not computer consoles in their bedrooms. Mean BMI in sample B was $18 \cdot 3[2 \cdot 0] \mathrm{kg} / \mathrm{m}^{2}$ (mean BMI centile $49 \cdot 1[25 \cdot 0]$ ) and mean $\%$ body fat was $13 \cdot 8[4 \cdot 1] \%$. Only 9 children $(5 \%)$ were classified as at risk of overweight according to IOTF cut-offs and none were classified as overweight. Significant differences were identified between countries with respect to BMI (centile), waist circumference and body fat $(p \leq 0 \cdot 001)$ - with the USA team having consistently and significantly greater measurements than their Chinese and South African counterparts $(p \leq 0 \cdot 02)$.

Global child overweight data for the 16 countries represented in sample B would predict a prevalence of overweight between 5.9\% and $37 \cdot 2^{\circ}{ }^{(3)}$. Therefore, whilst causal relationships cannot be confirmed, analysis of this unique international cohort suggests that habitual participation in team sport may be associated with other healthful behaviours and outcomes, not least a lower risk of overweight but also less screen time and screen access than would be predicted from comparable populations. With poor awareness of activity recommendations, further analysis of this and future events will focus on the motivations for sport participation and the means by which this can be communicated to currently inactive sub-groups.

1. WHO (2010) Global Recommendations on Physical Activity for Health. Switzerland: WHO Press.

2. OECD (2013) Health at a Glance 2013. Available: http://www.oecd.org/els/health-systems/Health-at-a-Glance-2013.pdf [Accessed March 2014].

3. IASO (2013) Global overweight in children Available: http://www.iaso.org/site_media/library/resource_images/Global_Childhood_Overweight_ October_2013.pdf [Accessed March 2014]. 